\title{
Background Firing Rates of Orbitofrontal Neurons Reflect Specific Characteristics of Operant Sessions and Modulate Phasic Responses to Reward-Associated Cues and Behavior
}

\author{
Alexxai V. Kravitz ${ }^{1}$ and Laura L. Peoples ${ }^{1,2}$ \\ ${ }^{1}$ Neuroscience Graduate Group, Department of Neuroscience, and 2Department of Psychiatry, University of Pennsylvania School of Medicine, Philadelphia, \\ Pennsylvania 19104
}

\begin{abstract}
The orbitofrontal cortex plays an important role in the ability of animals to adjust their behavior in response to behavioral outcomes. Multiple studies have demonstrated that responses of orbitofrontal neurons during operant sessions reflect the outcome of particular behaviors. These studies have focused on rapid neural responses to short-duration events such as instrumental behavior and rewardassociated discrete cues. We hypothesize that longer-lasting changes in firing are also important for information processing in the orbitofrontal cortex. In the present study, we recorded the activity of 115 single orbitofrontal neurons during a multiphase operant task in which the relationship between a lever-press response and a sucrose reward was varied between the different phases. Approximately one-half of the orbitofrontal neurons exhibited a change in background firing during the operant phases. These changes were observable across multiple behavioral and stimulus events and thus reflected a general shift in background firing. The majority of changes were selective for one or the other of the operant phases. Selective changes contributed to unique patterns of phasic firing time locked to cues and operant behavior in the two operant phases. These findings are consistent with the interpretation that changes in background firing of orbitofrontal neurons reflect operant session characteristics associated with behavioral outcome, and indicate further that changes in background firing contribute to the outcome selectivity of phasic firing patterns. More generally, we propose that the background firing rates of orbitofrontal neurons reflect contextual information, and facilitate context-appropriate event-related information processing and behavioral responses.
\end{abstract}

Key words: context; PFC; spontaneous; orbital; extracellular recording; neuronal ensembles; prefrontal

\section{Introduction}

The orbitofrontal cortex (OFC) plays an important role in the ability of animals to adjust their behavior according to changes in behavioral outcome and thus to maximize reward and minimize harm in a dynamic environment (Schoenbaum et al., 2002, 2003; Chudasama and Robbins, 2003; Schiller and Weiner, 2004; Pickens et al., 2005; Schoenbaum and Roesch, 2005; Boulougouris et al., 2007; Eagle et al., 2008; Pais-Vieira et al., 2007; Tait and Brown, 2007). Individual neurons in the rat OFC exhibit shortduration phasic responses time locked to operant session events such as instrumental behavior and reward-associated discrete cues. These phasic responses are characterized by the difference between "background" firing during a period before an event and "signal" firing during a period that includes the event. Phasic response magnitude varies with changes in the outcome associ-

Received Sept. 29, 2006; revised Dec. 13, 2007; accepted Dec. 14, 2007.

A.V.K. was supported by National Institute on Drug Abuse (NIDA) Grants DA-07241 (C. P. O'Brien) and DA-021449 (A.V.K.). This work was supported by NIDA Grants DA05186 (C. P. O'Brien) and DA012756 (H. Pettinati). Xun Wan, Karine Guillem, Jon Raksin, and Nick DeLong provided helpful comments on this manuscript.

Correspondence should be addressed to Laura L. Peoples, Department of Psychiatry, University of Pennsylvania, Transactional Research Laboratories, 125 South 31st Street, Philadelphia, PA 19104. E-mail: Ipeoples@psych.upenn.edu.

DOI:10.1523/JNEUROSCI.4344-07.2008

Copyright $\odot 2008$ Society for Neuroscience $\quad$ 0270-6474/08/281009-10\$15.00/0 ated with the signal period event. Outcome-selective phasic responses are thus hypothesized to be critical to the role of the OFC in facilitating flexible, outcome-directed behavior (Schoenbaum et al., 1999; Roesch and Olson, 2005; Gutierrez et al., 2006; Roesch et al., 2006, 2007; Stalnaker et al., 2006; van Duuren et al., 2007). Although previous electrophysiological studies of the OFC have focused on short-duration phasic responses, we hypothesize that longer-lasting changes in OFC firing are also involved in processing outcome-related information.

We recently reported that individual neurons in the nucleus accumbens exhibit significantly different background firing rates during different phases of a sucrose self-administration session (Kravitz et al., 2006). In the present experiment, we tested the hypothesis that changes in background firing can mediate certain types of information processing in the OFC (Peoples et al., 2004; Kravitz et al., 2006). Specifically, we predicted that changes in background firing would (1) be exhibited by OFC neurons during operant behavior, (2) vary with changes in operant behavioral outcome, and (3) contribute to phasic response variations associated with changes in operant response outcome.

To test these predictions, we recorded the activity of 115 single OFC neurons during a multiphase operant task in which the relationship between a lever-press response and a sucrose reward was varied between the different phases. The results of this study 
supported all three of our predictions. The findings reported here are thus consistent with the interpretation that changes in background firing of OFC neurons are associated with factors that vary with the behavioral outcome, and further that OFC background firing changes contribute to the outcome selectivity of phasic firing patterns. In conjunction with previous studies, our findings offer a novel view of information processing in reward-related brain structures: the background firing rates of individual neurons reflect specific long-lasting characteristics of the context and also modulate phasic neural responses time-locked to reward-associated cues and behavior. This may contribute to contextdependent information processing and behavior (Kravitz et al., 2006).

\section{Materials and Methods \\ Subjects}

Subjects were seven male Long-Evans rats. All animal care procedures and protocols were in accordance with the Guide for the Care and Use of Laboratory Animals published by the United States Public Health Service; procedures were also approved by the Institutional Animal Care and Use Committee of the University of Pennsylvania.

\section{Surgery and postoperative maintenance}

Animals were anesthetized with ketamine (30 mg/kg, i.p.) and xylazine (5 $\mathrm{mg} / \mathrm{kg}$, i.p.) before being placed in a stereotaxic apparatus. Anesthesia was maintained with isoflurane through a nose cone during surgery. One array of 16 Teflon-coated stainless-steel microwires was implanted unilaterally in the right $\mathrm{OFC}$ [anteroposterior, +2.2 to $+3.7 \mathrm{~mm}$; mediolateral, \pm 1.5 to $\pm 4.5 \mathrm{~mm}$; and dorsoventral, $-5 \mathrm{~mm}$ (Paxinos and Watson, 1996)]. A stainless-steel ground wire was also implanted under the skull. Rats weighed between 355 and $410 \mathrm{~g}$ before surgery and were maintained with food restriction at between 350 and $375 \mathrm{~g}$ ( $~ 85-90 \%$ of free-feeding weight) after surgery. Rats had ad libitum access to water.

\section{Apparatus}

All behavioral procedures were conducted in operant chambers purchased from Med Associates (St. Albans, VT). The chamber walls and floors were acrylic. Each chamber was equipped with a retractable lever, a signal light above the lever, a tone generator, a syringe pump for sucrose delivery, a drinking trough, and a house light (Med Associates). The chambers were enclosed inside sound-attenuating and ventilated cubicles.

\section{Behavioral procedures during training sessions}

Each training session consisted of three operant phases and three baseline periods (Fig. 1), presented in the following order: (1) a 30 min baseline period; (2) a 30 min operant phase in which lever-press responding was reinforced on a fixed-ratio 1 (FR1) schedule of primary sucrose reinforcement (referred to as the first sucrose reward phase); (3) a $60 \mathrm{~min}$ baseline period; (4) a 10 min operant phase in which lever presses were reinforced on an FR1 schedule of conditioned reinforcement (referred to as the conditioned reward phase); (5) a 30 min operant phase in which lever-press responding was again reinforced on an FR1 schedule of primary reinforcement (referred to as the second sucrose reward phase); and (6) a $30 \mathrm{~min}$ baseline period at the end of the experiment. The start of each operant phase was signaled by the illumination of a white house light and the insertion of a response lever. During the two sucrose reward phases, each reinforced lever press was followed immediately by delivery of sucrose to a drinking trough $(0.2 \mathrm{ml}$ of $32 \%$ sucrose delivered over
$10 \mathrm{~s}$ ), and a conditioned reinforcer consisting of the following: (1) a $10 \mathrm{~s}$ tone, (2) a $10 \mathrm{~s}$ illumination of a light above the lever, and (3) retraction of the lever. The response lever was reinserted $60 \mathrm{~s}$ after the lever-press response (period of lever retraction referred to as timeout). The conditioned reward phase was identical to the sucrose reward phase in all respects except that the sucrose delivery was omitted. During each baseline phase, the house light was turned off and the response lever was retracted from the chamber.

During training, the number of trials in the conditioned reward phase was progressively incremented until the animal could reliably complete 10 presses during the conditioned reward phase. Animals were trained for a minimum of $9 \mathrm{~d}$ (average $=10.8 \mathrm{~d}$ ) before the recording session.

Behavioral procedures during recording sessions

The session events during the recording sessions were identical to those of a daily training session except that additional probe and baseline phases were added to the end of the session. After the third baseline period, animals were exposed to three additional phases: (1) a $10 \mathrm{~min}$ "house light probe" period during which the chamber house light was illuminated, (2) a $30 \mathrm{~min}$ baseline period, and (3) a $30 \mathrm{~min}$ "sucroseprobe" period in which a Petri dish containing $40 \mathrm{ml}$ of $32 \%$ sucrose was placed into the chamber, so the animal could drink sucrose ad libitum. During the sucrose probe period, animals typically spent the first $10 \mathrm{~min}$ continuously drinking.

\section{Identification of start and end of drinking during the sucrose} reward operant phases

The start and the end of drinking during each trial in the first sucrose reward phase were identified to define "timeout" and "drinking" periods. The start of drinking was defined as the first infrared beam break after each lever-press response. The end of drinking was defined as the first beam exit that was followed by a period of at least $5 \mathrm{~s}$ without another beam break.

\section{Chronic extracellular recording procedures}

Voltage signals from each microwire were recorded, amplified (up to $32,000 \times$ ), processed, and digitally captured using commercial hardware and software (Plexon, Dallas, TX). Single units were discriminated offline with principal component analysis (Offline Sorter, Plexon). The quality of the recordings was ensured with the following criteria: (1) 
$<3 \%$ of all interspike intervals exhibited by the unit were shorter than $1000 \mu \mathrm{s}$, and (2) the average amplitude of the unit waveform was at least three times larger than that of the noise band.

\section{Analyses of neural firing}

All statistical analyses were performed in Excel (Microsoft, Redmond, WA) and Matlab (The MathWorks, Natick, MA). All mean results are presented as mean \pm SEM. For all analyses that used normalized values, firing rates were normalized with $(A-B) /(A+B)$, where $A$ and $B$ represent firing in the two periods that were compared. This normalization was used to compare firing changes in neurons with different overall firing rates, as well as to normalize the data for parametric statistics.

\section{Firing patterns}

Long-lasting changes in average firing rate. We tested each neuron for a long-lasting change in average firing rate during each operant phase relative to the preceding baseline phase. Firing rates were calculated in $30 \mathrm{~s}$ bins for the entire experiment, and statistical comparisons were performed using a Mann-Whitney test ( $\alpha=0.01$ ). Additionally, for each $30 \mathrm{~s}$ bin during each phase, we determined whether firing rate was higher or lower than for the preceding baseline. Only neurons that exhibited a significant change, as well as $>90 \%$ of the bins above (for increases) or below (for decreases) the average firing rate of the baseline, were defined as showing long-lasting changes in average firing rate.

Changes in background firing rate. We performed additional analyses to test the stability of firing rate changes across multiple behavioral events during the session. We calculated the average firing rate of all recorded neurons in the following behavioral periods: press $(1-0 \mathrm{~s}$ before the press), cue ( $0-10 \mathrm{~s}$ after the press), drinking ( $10-25 \mathrm{~s}$ after the press), and timeout (40-55 s after the press). During the drinking period, the rat spent the entire time at the drinking trough, with no locomotion; during the timeout period, the rat exhibited high levels of locomotion and no drinking. Neurons that exhibited both a significant change in average firing rate during the operant phase and a $>20 \%$ change in firing rate during both the drinking and timeout periods (relative to the preceding baseline period) were defined as exhibiting a change in background firing. We tested whether these groups of neurons exhibited significantly different firing in all four behavioral periods (press, cue, drinking, and timeout). We analyzed the normalized data with repeated-measures ANOVA, using individual paired $t$ tests for post hoc comparisons.

Long-lasting changes in average firing rate during the probe phases. We tested each neuron for a long-lasting change in average firing rate during the drinking and the house light probe phases at the end of the session. Firing rates were calculated in $10 \mathrm{~s}$ bins for the first $10 \mathrm{~min}$ of each probe phase and for the $10 \mathrm{~min}$ of baseline that preceded each probe phase. Firing during the probe and its preceding baseline were compared using a Mann-Whitney test $(\alpha=0.01$ ). Additionally, for each $10 \mathrm{~s}$ bin during each phase, we determined whether firing was higher or lower than for presession baseline. Only neurons that exhibited both a significant change and had $>90 \%$ of the bins above (for increases) or below (for decreases) the average firing rate of the first baseline phase were defined as having undergone long-lasting changes in average firing rate during the probe phase.

Phasic changes in firing time locked to the lever-press operant. We tested each neuron for phasic changes in firing time locked to the lever-press response in each phase. The first 10 trials of each phase were used for this analysis, because the conditioned reward phase included only 10 trials. Firing rates were calculated on a trial-by-trial basis during a $1 \mathrm{~s}$ period of background firing ( $6-5 \mathrm{~s}$ before the press), and a $1 \mathrm{~s} \mathrm{period} \mathrm{of} \mathrm{signal} \mathrm{firing}$ before or after the conclusion of the press. The $1 \mathrm{~s}$ period before the press included the approach to the lever and the lever press itself. The $1 \mathrm{~s}$ period after the press included the onset of the conditioned cues and locomotion toward the drinking trough. The firing rates during the background and signal periods were compared with Wilcoxon matched pairs tests $(\alpha=$ $0.01)$. Significant changes in the prepress period were referred to as phasic lever-press responses, and significant changes in the postpress period were referred to as phasic cue responses.

Changes in phasic responses between the conditioned reward phase and the second sucrose reward phase. Phasic response amplitude differences between the conditioned reward phase and the second sucrose reward phase were calculated as the difference in normalized amplitude in each phase. The background and signal windows used to calculate phasic response magnitudes were the same as those used to identify lever-press and cue responses as described above. Differences in signal and background firing rates were calculated as the normalized difference in firing between these windows in each phase. Changes in lever-press and cue responses were analyzed separately and then combined for the regression analysis presented in Figure 10. In this analysis, there were eight neurons that exhibited both a cue response and a press response. The phasic responses of these neurons were calculated as the averages of their press and cue responses so that each neuron contributed only one data point to the regression. Similar results were obtained when the analysis was repeated independently for the press responses and cue responses.

\section{Histology}

Rats were injected with a lethal dose of ketamine solution $(200 \mathrm{mg} / \mathrm{kg}$, i.p.) and xylazine (30 mg/kg, i.p.). Anodal current (50 $\mu \mathrm{A}$ for $5 \mathrm{~s}$ ) was passed through each microwire. Animals were perfused with $\sim 300 \mathrm{ml}$ of $4 \%$ paraformaldehyde in $0.9 \%$ saline and $\sim 50 \mathrm{ml}$ of a solution of $5 \%$ potassium ferricyanide and $10 \%$ hydrochloric acid to stain the iron deposits left by the recording tips. The brains were cut into $50 \mu \mathrm{m}$ coronal sections, which were mounted on slides. Each slide was photographed, and the location of each wire tip was plotted on the coronal plate that most closely corresponded to its anterior-posterior position. Neurons recorded from wires that were not within the boundaries of the OFC were excluded from this experiment.

\section{Results}

\section{Behavior}

Operant behavior was contrasted across the conditioned reward operant phase and the two sucrose reward phases (Fig. 1) of the session. Lever-press behavior was similar across all three operant phases of the recording session. Average response rates during the first and second sucrose reward phases equaled $0.98 \pm 0.01$ and $0.98 \pm 0.01 \mathrm{press} / \mathrm{min}$, respectively. During the intervening conditioned reward phase, average response rate was $0.92 \pm 0.03$ presses/min. These rates were not significantly different (ANOVA: $\left.F_{(2,18)}=3.2, p>0.05\right)$ and approximated the maximal possible rate of 1 press $/ \mathrm{min}$.

\section{Neuron sample}

One hundred fifteen OFC neurons were recorded in this experiment. The average amplitude of recorded neurons equaled $184.6 \pm 7.6 \mu \mathrm{V}$. The average number of neurons recorded per rat was $16.4 \pm 3.8$. Histology is shown in Figure 2. There were no observed differences between groups of neurons recorded from the medial or lateral OFC for any of the main analyses (data not shown).

\section{Prediction 1: long-lasting changes in background firing rate are exhibited by OFC neurons}

Long-lasting changes in average firing during the first sucrose reward phase

We observed long-lasting changes in average firing during the first sucrose reward phase (Fig. 3). Across the population, 20 (17.4\%) neurons exhibited an increase in firing and $15(13.0 \%)$ exhibited a decrease in firing.

\section{Long-lasting changes in average firing are observable across multiple behavioral events}

The changes in average firing could have reflected either (1) a change in firing during one particular behavioral event during the session or (2) a more general change in firing (i.e., a change in background firing) that is observable across multiple behavioral events. We found the latter to be the case. Each trial of the first 
sucrose reward phase included the following four behavioral periods: (1) the lever press, (2) a postpress cue period, (3) a drinking period, and (4) a timeout period. The drinking period (10-25 s after the press) was characterized by sustained drinking. Conversely, the timeout period (40-55 s after the press) was characterized by high levels of locomotion and no drinking. Average firing rates during these periods were not necessarily equal (Fig. 4A). Nevertheless, 18 (90\%) of the neurons that exhibited an increase in average firing also exhibited increased firing rates during both the timeout and the drinking periods relative to the presession baseline period (Fig. $4 B$ ). Similarly, 12 (80\%) of the neurons that exhibited a decrease in average firing also exhibited decreased firing during both of these periods relative to the presession baseline (Fig. 4A). Neurons that exhibited a change in average firing as well as a consistent change in the timeout and drinking periods relative to baseline are henceforth referred to as neurons that exhibited "changes in background firing."

Group mean analyses of neurons that
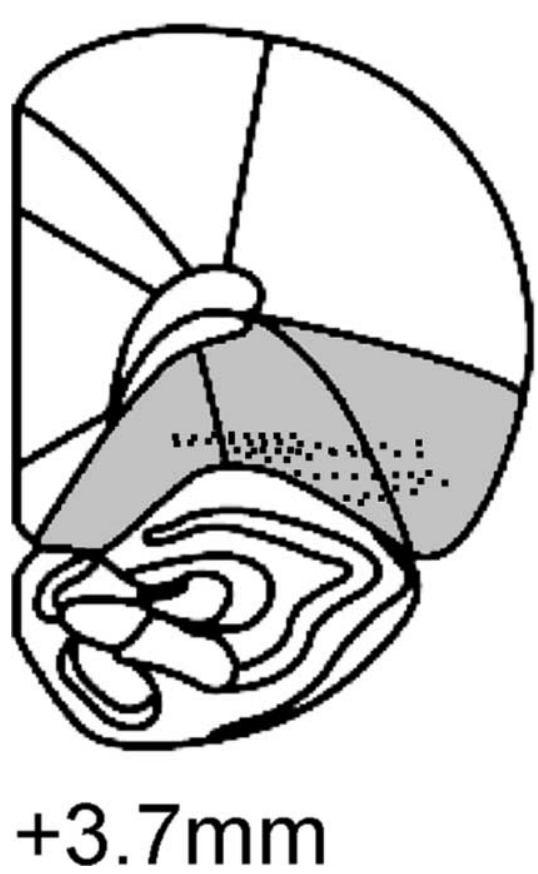

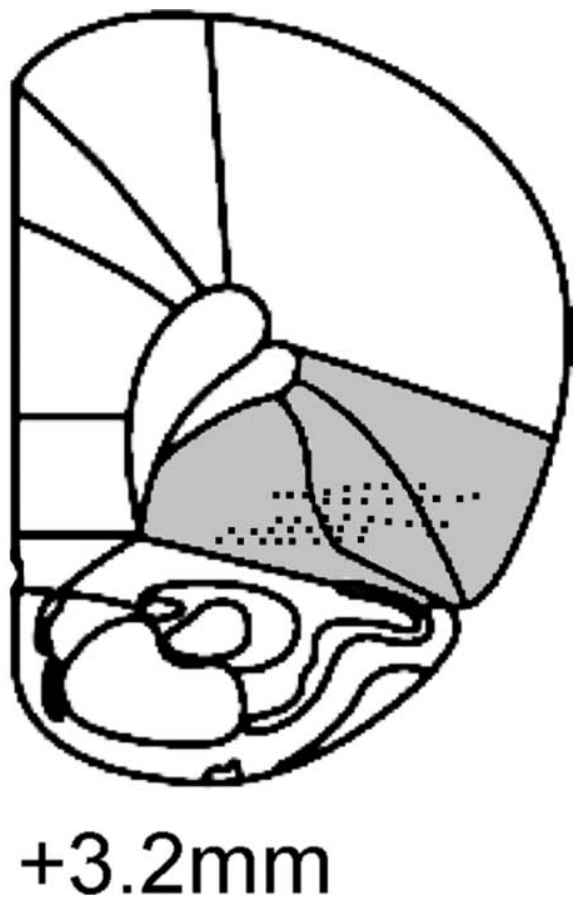

Figure 2. Histology. Locations of individual wire tips in the OFC (shaded regions) are presented on coronal sections. Numbers indicate millimeters anterior to bregma. exhibited changes in background firing showed that these neurons exhibited a consistent shift in average firing during all behavioral periods of the operant phases relative to their firing during the presession baseline. We applied a onefactor (behavioral period: baseline, press, cue, drinking, and timeout) repeated-measures ANOVA to analyze the normalized firing rates of the 18 neurons that exhibited an increase in background firing. There was a significant effect of behavioral period on firing rate (Fig. 5) $\left(F_{(4,14)}=12.3, p<0.001\right)$. Post hoc tests (paired $t$ tests) showed that firing during each of the four periods was significantly elevated relative to baseline $(p<0.025)$. We performed the same analysis on the 12 neurons that exhibited a decrease in background firing rate. Again, there was a significant effect of behavioral period on firing rate (Fig. 5) $\left(F_{(4,8)}=11.1\right.$, $p<0.005$ ). Post hoc tests (paired $t$ tests) showed that firing during each of the four periods was significantly decreased, relative to baseline $(p<0.005)$. Based on these findings it can be concluded that changes in average firing rate during the session were not attributable to any specific behavioral event, but rather reflected a more general background firing change that was observable across multiple behavioral events during the session.

Prediction 2: long-lasting changes in background firing rate reflect specific characteristics of the operant session

Long-lasting changes in firing were dissimilar between dissimilar operant phases

Changes in background firing similar to those observed during the first sucrose reward phase were also observed during the conditioned reward and second sucrose reward phases. During the conditioned reward phase, 20 neurons (17\%) showed an increase in background firing and 7 neurons $(6 \%)$ showed a decrease. During the sucrose reward phase, 18 (16\%) and 12 (10\%) neurons showed an increase and decrease in firing, respectively. The prevalence of increases and decreases in background firing did not differ significantly across the three operant phases $\left(\chi_{(2)}^{2}=\right.$ $1.6, p=0.44)$. Overall, $53(46 \%)$ of the recorded neurons exhib-

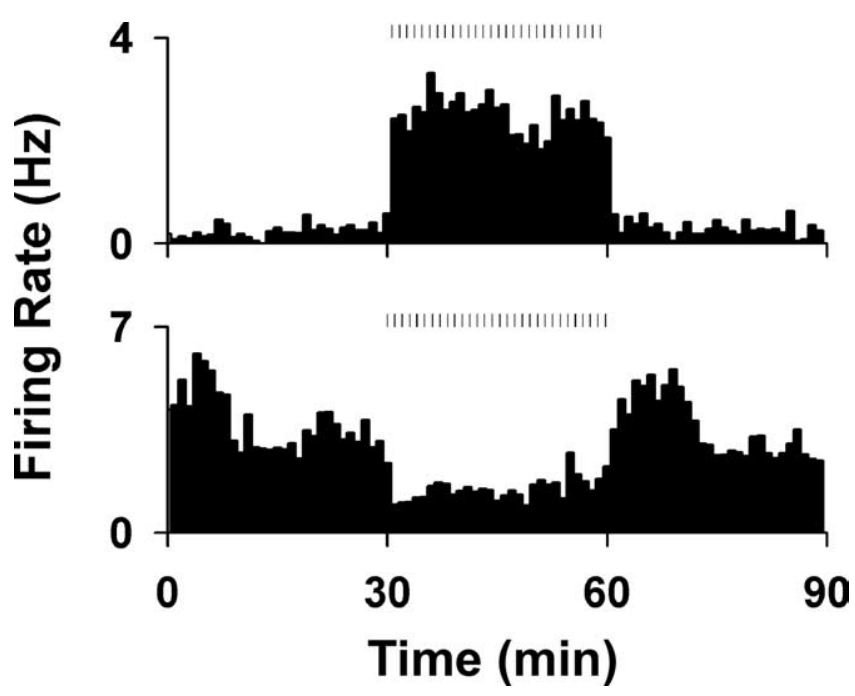

Figure 3. Changes in average firing rate during the first sucrose reward phase. Examples of changes in firing rates (in hertz) of two neurons during the first sucrose reward phase. The phase started at time $=30 \mathrm{~min}$ and lasted until time $=60 \mathrm{~min}$. The top histogram shows an example of an increase in average firing rate, and the bottom shows an example of a decrease. Ticks above the histograms represent lever-press responses during the phase.

ited a change in background firing during at least one phase of the experiment. Based on the responses during the conditioned phase and the second sucrose reward phase, neurons were classified into three response types: neurons that exhibited a change in background firing solely during the sucrose reward phase, neurons that exhibited a change in background firing solely during the conditioned reward phase, and neurons that exhibited a change in background firing during both of these phases (Fig. 6). Twenty-six (23\%) neurons exhibited an increase in background firing selectively during either the sucrose reward phase or the conditioned reward phase, whereas only 6 (5\%) neurons exhib- 


\section{A}
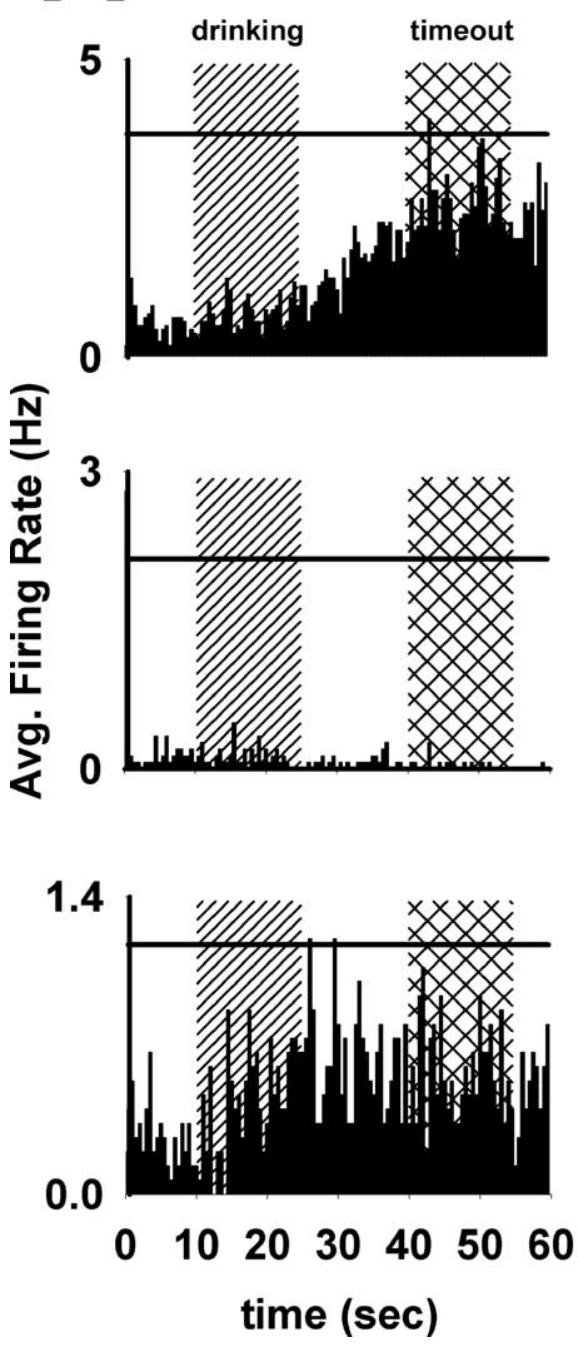

B

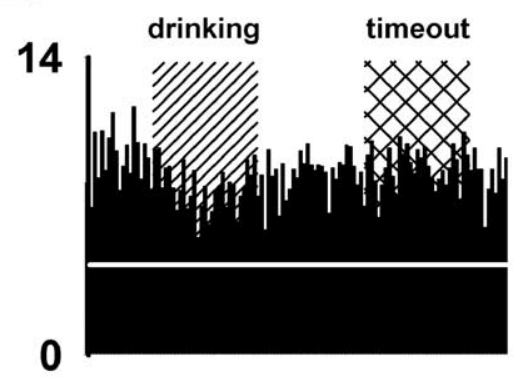

50

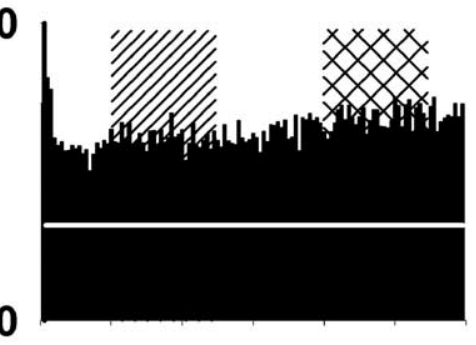

14
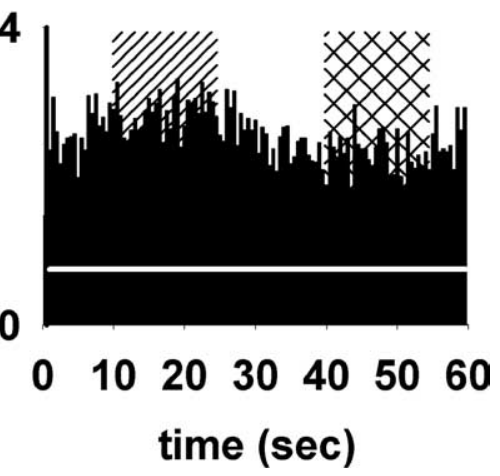

Figure 4. Examples of changes in background firing rate. $\boldsymbol{A}$, Three examples of neurons that exhibited decreases in background firing rate during the operant phase. These plots show perievent histograms of the 30 trials in the first sucrose reward phase from the completion of the lever-press response (time $=0 \mathrm{~s}$ ) to the reentry of the lever (time $=60 \mathrm{~s}$ ). The drinking period $(10-25 \mathrm{~s}$ after the press) and the timeout period ( $40-55 \mathrm{~s}$ after the press) are represented by vertical background bars. Horizontal lines represent the average firing rate of each neuron during the baseline period preceding the operant phase. $\boldsymbol{B}$, Three examples of neurons that exhibited increases in background firing rate during the operant phase, with plot parameters identical to those in $\boldsymbol{A}$.

ited an increase in both phases $(z$ test, $z=3.8, p<0.001)$. Similarly, 15 (13\%) neurons exhibited a decrease in background firing selectively during either the sucrose reward phase or the conditioned reward phase, whereas only $2(2 \%)$ neurons exhibited a decrease in both phases ( $z$ test, $z=3.3, p<0.001$ ). These findings show that the majority of the changes in background firing were selective for one or the other of the operant phases.

Long-lasting changes in firing were similar between similar operant phases

Seventy-three percent of the neurons that exhibited a change in background firing in the second sucrose reward phase exhibited a directionally consistent change in the first sucrose reward phase. In contrast, only $35 \%$ of these same neurons exhibited a directionally consistent change in the conditioned reward phase $(z=$ $2.8, p<0.01)$. Consistent with this finding, there was a stronger correlation between the changes in firing rate of all recorded neurons during the two sucrose reward phases (Fig. 7A) than between the changes during the first sucrose reward phase and the conditioned reward phase (Fig. $7 B$ ) (ANCOVA: $F_{(1,226)}$ $>7.0, p<0.01)$. These analyses support the conclusion that changes in background firing were stable across repetitions of the same type of phase (first and second sucrose reward phases).

Correlates of changes in background firing As described above, changes in background firing were apparent across different behavioral periods (e.g., timeout, press period, cue period, and drinking period), which involved different stimuli and behavioral events. The changes in background firing thus did not reflect an effect of short-duration neural responses on average firing. We considered various factors unique or common to the two types of operant phases that could have contributed to changes in background firing. These are discussed separately for the neurons that showed phase-selective and nonselective changes in background firing.

Selective changes in firing during either the sucrose reward phase or the conditioned reward phase. The changes in background firing that were selective either for the sucrose reward phase or for the conditioned reward phase would be expected to vary with factors unique to each phase. Variables that differentiated the two phases included operant behavioral outcome and exposure to the primary sucrose reward. Specifically, operant behavior was followed only by conditioned cues during the conditioned reward phase but was followed by both conditioned cues and primary reward during the sucrose reward phases. To test for a possible relationship between background firing change and contact with the primary reward, we exposed animals to an additional probe phase after the last operant phase. During the probe phase, animals had ad libitum access to sucrose in the absence of the lever and other cues associated with the operant phases. Thirty-two percent of the neurons that exhibited a selective change in background firing during the sucrose reward phase also showed a similar change in average firing when the animal engaged in a sustained period of sucrose consumption.

The findings of the sucrose probe phase indicate that approximately one-third of the changes in background firing selective for the sucrose reward phase were potentially associated with exposure to the primary reward. Based on this association, the long-lasting change in firing during the sucrose reward phase could have reflected a number of factors. First, the changes in firing during the sucrose reward phase could have reflected a neural response to drinking per se. By definition, however, it could not be the case that all of the background firing change was attributable to drinking alone. Second, the changes in background firing during the sucrose reward phase could have re- 


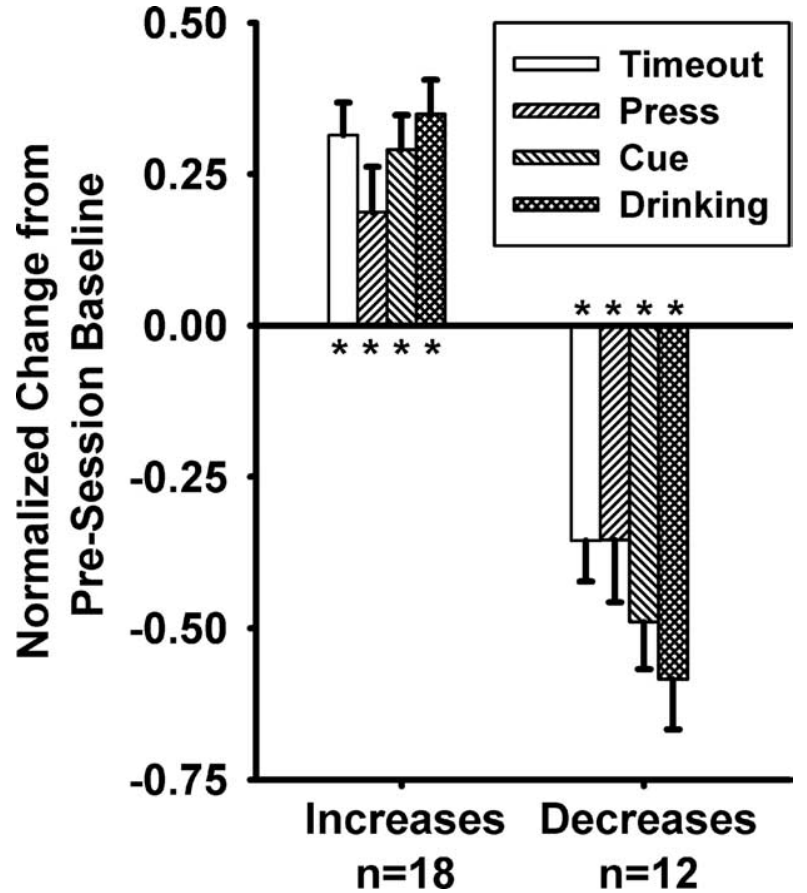

Figure 5. Firing rate changes during the sucrose reward phase were directionally consistent across behavioral periods. Normalized change from baseline during the press, cue, drinking, and timeout periods are represented on the $y$-axis. Normalized firing rates equal $(A-B) /(A+B)$ where $A$ equals the average firing rate within the behavioral period, and $B$ equals the average firing within the baseline period. Data are presented for all neurons that exhibited an increase in background firing (left bars) and for all neurons that exhibited a decrease in background firing (right bars). Asterisks indicate $p<0.025$. Error bars represent the SEM percentage change.

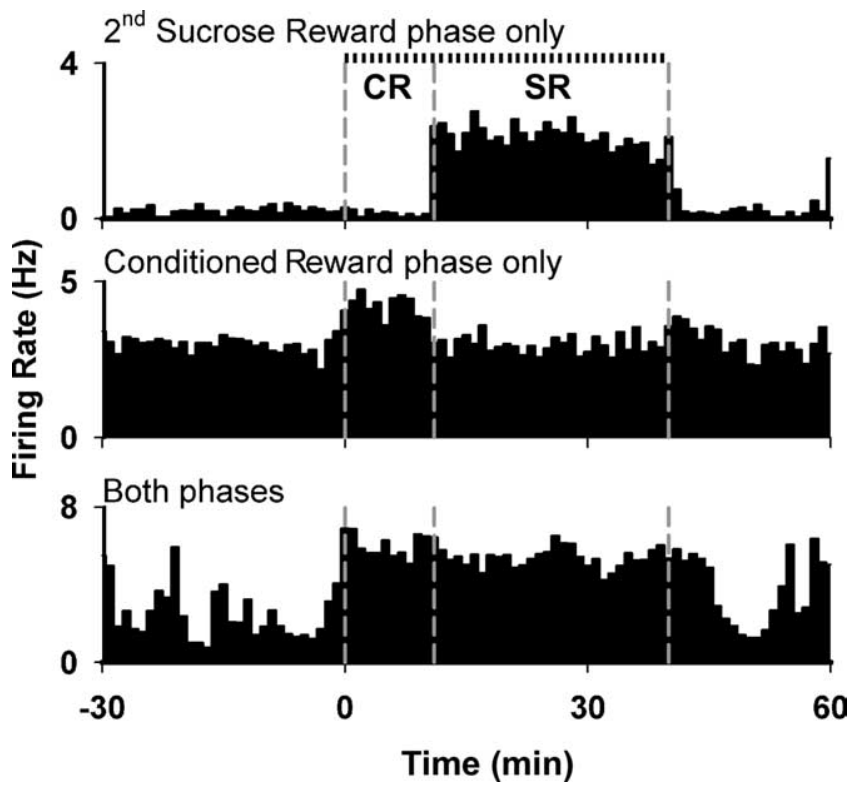

Figure 6. Examples of the three response types simultaneously recorded from the same rat during the conditioned reward phase (CR) and the second sucrose reward phase (SR). Vertical dashed lines represent (from left to right) the start of conditioned reward phase, the start of the sucrose reward phase, and the end of the sucrose reward phase. Tick marks above the top histogram represent lever presses.

flected the co-occurrence of exposure to primary reward and either the operant response or a conditioned stimulus. However, this is unlikely, because the sucrose probe occurred outside the operant task and in the absence of conditioned cues typically
A
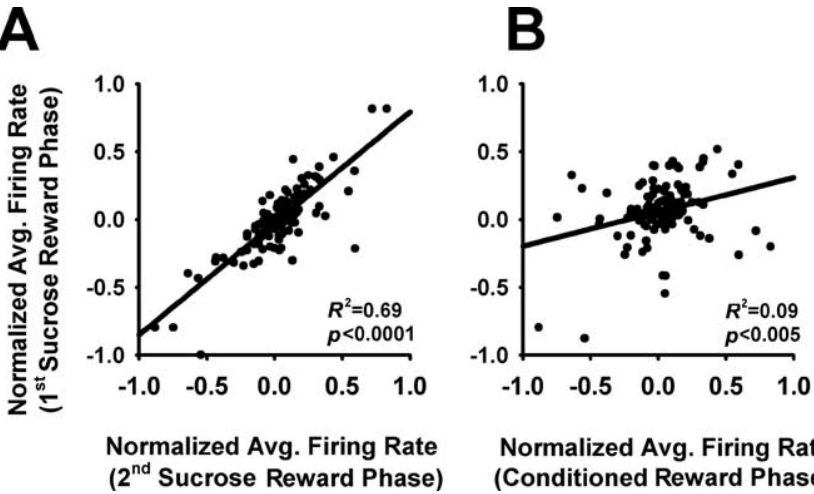

Normalized Avg. Firing Rate (Conditioned Reward Phase)

Figure 7. Correlations between average firing during the first sucrose reward phase and either the second sucrose reward phase or the conditioned reward phase. $A$, Correlations between the normalized average firing rates of all recorded neurons in the two sucrose reward phases. Normalized firing rates equal $(A-B) /(A+B)$, where $A$ equals the average firing rate within the operant phase, and $B$ equals the average firing within the preceding baseline period. Lines represent linear regressions, and $R^{2}$ and $p$ values of the regressions are presented on each plot. $\boldsymbol{B}$, Correlations between the normalized average firing rates of all recorded neurons in the first sucrose reward phase and the conditioned reward phase. All plot parameters are as in $\boldsymbol{A}$.

associated with the availability and delivery of sucrose reward. A third possibility is that the neural responses during the sucrose probe, and changes in background firing by the same neurons during the sucrose reward phase, could have reflected a response to the incentive value of the sucrose reward. This latter possibility is consistent with the characteristics of OFC neuronal phasic responses to primary reward (Tremblay and Schultz, 1999).

The findings of the sucrose probe phase indicate that the majority of phase-selective changes in background firing reflect something other than factors associated with exposure to the primary reward. An alternative factor that differentiated the conditioned reward and sucrose reward phases was the outcome of the operant response. Thus, it is possible that the phase selectivity of the long-lasting changes in background firing reflected a between-phase difference in the expected outcome of the operant. A relationship between background firing and outcome expectancy (i.e., expected consequence of a particular act) (Schoenbaum and Roesch, 2005) would be consistent with observed characteristics of phasic OFC firing patterns (see Discussion).

Nonselective changes in background firing during the sucrose reward phase and the conditioned reward phase. Nonselective changes in background firing were expected to reflect shared characteristics of the conditioned reward phase and the sucrose reward phase. Variables common to these phases included reward-directed behavior and reward-associated conditioned stimuli. Although short-duration neural responses to those events cannot wholly explain the changes in background firing, it was possible that firing patterns were related to sustained states associated with the events.

We tested for a relationship between background firing change and one conditioned cue that was common to the conditioned reward and sucrose reward phases, the illumination of the house light. Animals were exposed to a probe phase at the end of the experiment. During the probe phase, the house light was illuminated for $10 \mathrm{~min}$ in the absence of the lever, sucrose, or any other cues associated with the operant phase. Thirty-eight percent of the neurons that exhibited a sustained change in background firing across the conditioned reward phase and the sucrose reward phase also showed a similar change when animals were exposed to the house light alone. This indicates that some of 
Table 1. Prevalence of phasic responses in the press and cue periods

\begin{tabular}{|c|c|c|c|c|c|c|}
\hline & \multicolumn{2}{|c|}{ Conditioned reward phase } & \multicolumn{2}{|c|}{ Second sucrose reward phase } & \multicolumn{2}{|c|}{ Both phases } \\
\hline & Increase & Decrease & Increase & Decrease & Increase & Decrease \\
\hline Press & $14(12 \%)$ & $6(5 \%)$ & $9(8 \%)$ & $8(7 \%)$ & $5(4 \%)$ & $4(3 \%)$ \\
\hline Cue & $13(11 \%)$ & $8(7 \%)$ & $14(12 \%)$ & $7(6 \%)$ & $2(2 \%)$ & $2(2 \%)$ \\
\hline
\end{tabular}

Prevalence of phasic responses were determined separately for press and cue responses. Some neurons exhibited both a press and a cue response and wer included in both groups.

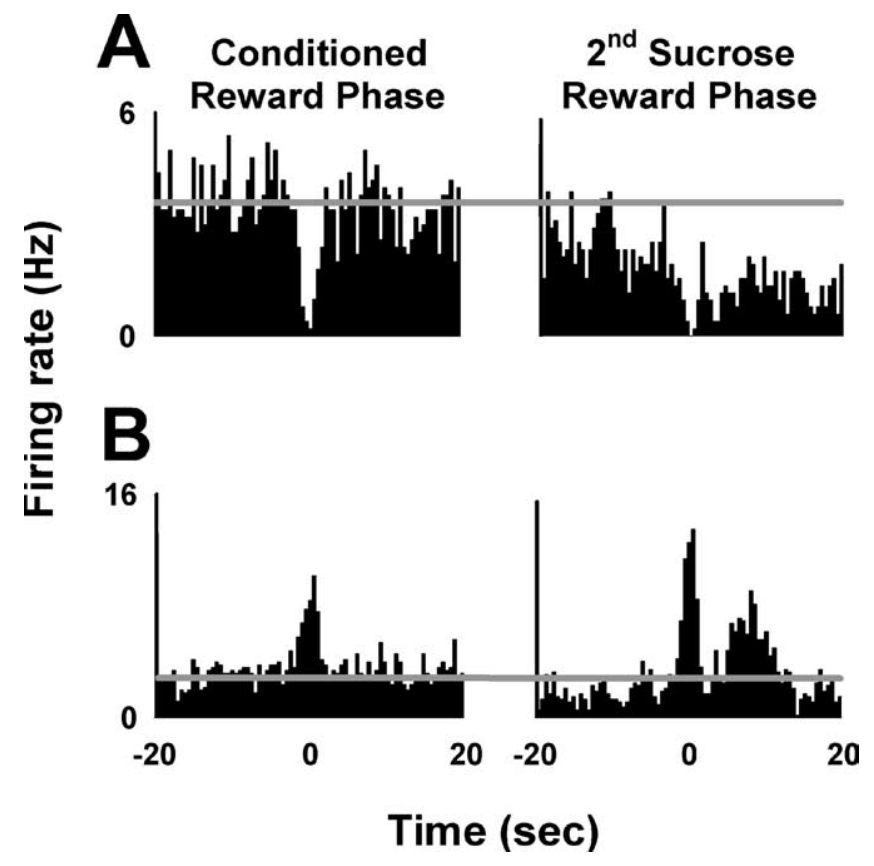

Figure 8. Two example neurons representing phasic firing rate changes. $\boldsymbol{A}$, Firing rate decrease from one neuron presented for the conditioned reward phase (left) and the sucrose reward phase (right). Lever-press response occurred at time $=0$. Firing rate in hertz is plotted as a function of time in $0.5 \mathrm{~s}$ intervals from -20 to $+20 \mathrm{~s}$ before and after the lever press. Horizontal lines mark the average background firing rate during the conditioned reward phase. $\boldsymbol{B}$, Firing rate increase from one neuron. All plot parameters are as in $\boldsymbol{A}$.

the nonselective changes we observed in background firing may have reflected either the sensory or associative characteristics of the house light cue. However, the majority of the phase nonselective changes in background firing were not related to the house light cue.

Prediction 3: long-lasting changes in background firing rate influence the magnitude of event-related responses

Lever-press responses in the conditioned reward phase and the second sucrose reward phase

Forty-two neurons exhibited a phasic response to either or both the lever-press response and the onset of the cue period (Table 1). Thirty-four ( $81 \%$ ) of these 42 neurons exhibited a $>20 \%$ change in phasic response amplitude between the conditioned reward phase and the sucrose reward phase.

For $13(38 \%)$ of these 34 neurons, the phasic response decreased in amplitude between the conditioned reward and sucrose reward phases (mean magnitude of decrease $=93 \pm 9 \%$ ). Consistent with this decrease, the phasic response was statistically significant for all 13 of these neurons during the conditioned reward phase but for only 4 of the 13 neurons during the sucrose reward phase. An example of a neuron that exhibited a phasic decrease that was weakened (i.e., amplitude decreased) in the sucrose reward phase is presented in Figure $8 \mathrm{~A}$.
For $21(62 \%)$ of the 34 neurons, the phasic response increased in amplitude between the conditioned reward and sucrose reward phases (mean magnitude of increase $=152 \pm 12 \%$ ). Consistent with this increase, the phasic response was statistically significant for 8 of these neurons during the conditioned reward phase, but for all 21 during the sucrose reward phase. An example of a neuron that exhibited a phasic increase that was strengthened (i.e., increased in amplitude) during the sucrose reward phase relative to the conditioned reward phase is shown in Figure $8 B$.

Despite the changes in the phasic response of individual neurons, there were no significant differences in the overall prevalence or magnitude of phasic responses between the conditioned reward and sucrose reward phases. Additionally, there were no between-phase differences in the prevalence and average amplitude of either phasic increases or phasic decreases. These analyses indicate that the overall prevalence and magnitude of phasic responses between the two phases were similar, but that different groups of neurons exhibited phasic responses in each phase.

The contribution of signal and background firing to changes in the amplitude of phasic responses between the two phases

To understand the relative contributions of signal and background firing to the between-phase changes in phasic response amplitudes, we calculated the magnitude of the change between the two phases in the 34 neurons that exhibited a $>20 \%$ change in phasic response amplitude between the two phases. Twenty-two of these neurons exhibited phasic increases, whereas 12 exhibited phasic decreases. For the 22 neurons that exhibited phasic increases, changes in phasic response magnitude were positively correlated with changes in signal firing and were negatively correlated with changes in background firing between the phases (Fig. 9A). For the 12 neurons that exhibited phasic decreases, phasic response magnitude changes were negatively correlated with changes in signal firing and were positively correlated with changes in background firing between the phases (Fig. 9B). In addition, we found that the changes in signal and background firing of individual neurons were inversely correlated for the 34 neurons that exhibited a $>20 \%$ change in phasic amplitude between the phases (Fig. 10). In other words, neurons that exhibited increases in signal firing most often exhibited decreases in background firing, and vice versa. This inverse relationship is associated with changes that increase, as well as changes that decrease, the amplitude of phasic responses. For example, in Figure $8 B$, the phasic increase gets stronger because of both an increase in signal and a decrease in background (as for neurons represented in the top left quadrant of Fig. 10). If instead the signal had decreased and the background had increased (as in the bottom right quadrant of Fig. 10), the amplitude of the phasic response would have been weakened.

\section{Discussion}

We present three major findings: (1) OFC neurons exhibit changes in background firing rate during periods of operant behavior; (2) these changes vary with changes in the outcome of the operant and other specific characteristics of the operant task; and (3) changes in background firing contribute to variations in phasic responses that occur with changes in the outcome of operant behavior. 

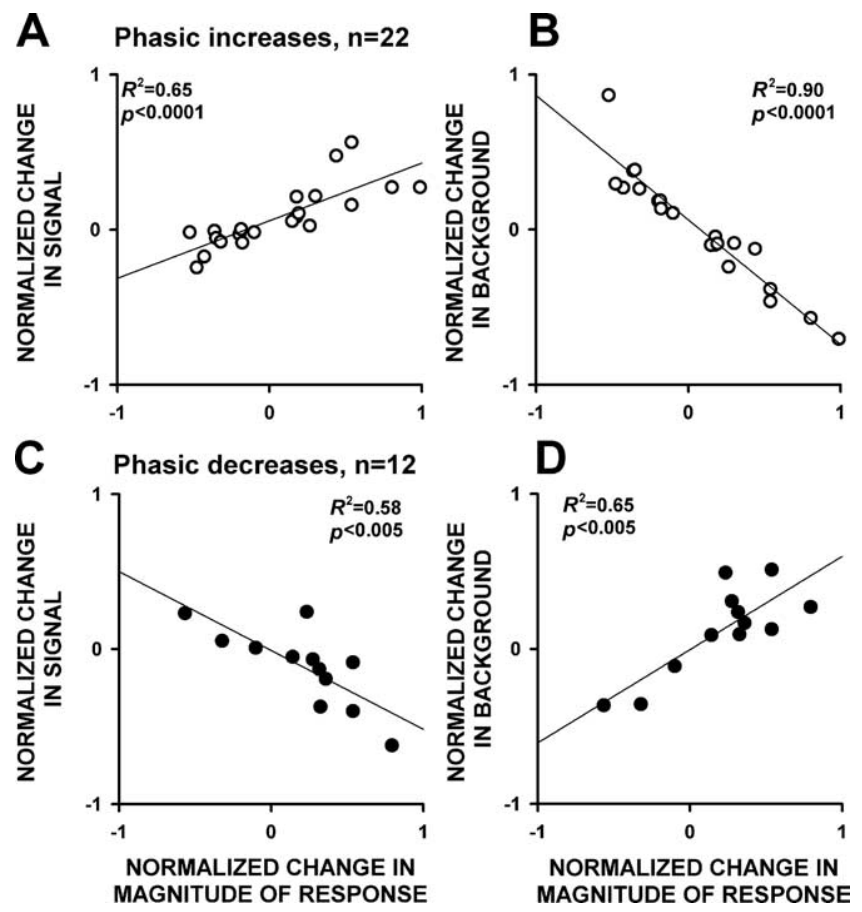

Figure 9. Relative contribution of signal and background to changes in phasic response magnitude between the conditioned reward phase and the sucrose reward phase. $A$, Correlations between the change in signal firing and the change in the magnitude of phasic increases that occurred between the conditioned reward phase and the sucrose reward phase. $\boldsymbol{B}$, Correlations between the change in background firing and the change in the magnitude of phasic increases that occurred between the conditioned reward phase and the sucrose reward phase. Positive values for the change in the magnitude of the response correspond to a strengthening (increase in amplitude) of the phasic increase. Lines represent linear regressions, and $R^{2}$ and $p$ values are presented on the plots. $\boldsymbol{C}, \boldsymbol{D}$, The same information is given for the neurons that exhibited phasic decreases. Positive values for the change in the magnitude of the response correspond to a strengthening (increase in amplitude) of the phasic decreases.

\section{OFC neurons exhibit long-lasting changes in}

background firing

Approximately one-half (46\%) of the recorded OFC neurons exhibited changes in background firing during at least one operant phase of the experiment. These changes were not attributable to any one particular behavior, but instead reflected a general change in firing rate across multiple behavioral events. Similar long-lasting changes in firing rate have now been observed in two reward-related structures (accumbens and OFC), raising the possibility that such changes may occur throughout reward-related brain circuitry (Kravitz et al., 2006).

Long-lasting changes in background firing rate reflect specific characteristics of the operant phases

Studies of OFC firing patterns during operant sessions have shown that subsets of neurons exhibit phasic responses time locked to different events during the session, including the operant behavior, outcome-associated conditioned cues, and the outcome of the operant behavior. The majority of the responses to the cues and operant behavior are selective for the particular outcome associated with those events. Similarly, the majority of responses to reward tend to be selective for that particular reward. A minority of the phasic OFC firing patterns are outcome nonselective and thus may reflect sensory or task-related characteristics of the event (Schoenbaum et al., 1999; Tremblay and Schultz, 1999; Schultz et al., 2000; Roesch and Olson, 2005; Gutierrez et al., 2006; Roesch et al., 2006, 2007; Stalnaker et al., 2006;

\section{Phasic changes, $n=34$}
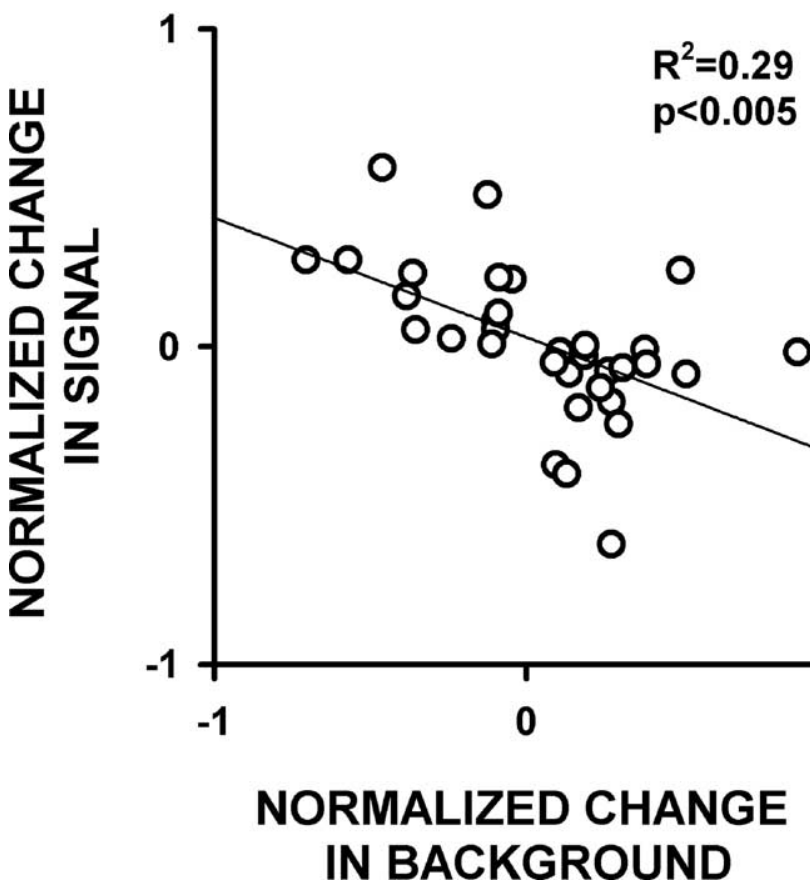

Figure 10. Normalized changes in signal and background firing between the conditioned reward phase and the sucrose reward phase are inversely correlated. The line represents a linear regression, and $R^{2}$ and $p$ value are presented on the plot.

Hosokawa et al., 2007; Simmons and Richmond, 2008; van Duuren et al., 2007). The background firing changes observed in the present study exhibited a number of characteristics consistent with those of phasic OFC firing patterns. For example, subsets of neurons showed changes in background firing that were associated with particular aspects of the operant session, including the primary sucrose reward and the reward-associated house light cue. More importantly, the majority $(84 \%)$ of the changes in background firing were selective for operant phases that were highly similar except for the outcome of the operant response. Although the exact correlates of these changes have not been conclusively identified, our findings are consistent with the interpretation that changes in background firing might reflect some of the same factors that influence phasic OFC firing patterns.

\section{Long-lasting changes in background firing influence} phasic responses

The difference in background firing between the conditioned reward phase and the sucrose reward phase was associated with changes in the magnitude of phasic lever-press responses between the phases. It has been reported previously that variations in the outcome of events can alter the magnitude of phasic responses to those events (Schoenbaum et al., 1999, 2006; Schoenbaum and Roesch, 2005; Stalnaker et al., 2006; Hosokawa et al., 2007; Simmons and Richmond, 2008). In the present experiment, we have demonstrated that changes in both background and signal contribute to these changes in phasic responses.

The observation that background firing changes modulate phasic responses to discrete events is in agreement with observations of other researchers. In the visual cortex of an anesthetized cat, neuronal activity (measured optically with voltage-sensitive dye, concurrent local field potentials, and single-unit microelectrode recordings) exhibited spontaneous fluctuations in back- 
ground that explained most of the trial-to-trial variability in the magnitude of visually evoked neural responses (Arieli et al., 1996). The authors of that study proposed that background activity "may provide the neuronal substrate for the dependence of sensory information processing on context and on behavioral and conscious states." A similar conclusion was reached based on event-related fluctuations in human blood oxygen leveldependent responses, where spontaneous fluctuations accounted for a significant fraction of trial-to-trial variability (Fox et al., 2006). In addition, changes in both signal and background have been shown to contribute to satiety-related changes in single neuron responses in the primate OFC (Critchley and Rolls, 1996). Finally, pharmacological manipulations that change the background activity of single neurons in the accumbens have been linked to changes in event-related neuronal responses (Rolls et al., 1984; Kiyatkin and Rebec, 1996; Peoples and Cavanaugh, 2003, Peoples et al., 2007). The present investigation is in agreement with these studies, but also demonstrates that background activity does more than modulate the magnitude of event-related phasic responses: it also reflects specific characteristics of the operant context. This could contribute to long-lasting, contextdependent regulation of neural responses to events.

\section{Conclusion and implications}

The present study provides new information regarding the neurophysiology of the OFC. Individual neurons in the OFC exhibit changes in background firing during periods of operant behavior. These changes reflect specific characteristics of operant tasks, perhaps including response outcomes and other factors that normally influence phasic OFC firing patterns. Additionally, in this study we observed that background firing changes contributed to outcome selectivity of phasic responses. Changes in signal and background firing tended to vary inversely such that certain phasic responses were enhanced and others were weakened. The present findings do not provide information about the mechanisms that might mediate the inverse relationship between changes in background and signal firing. However, this inverse relationship is reminiscent of that which appears to exist between tonic extracellular levels of certain neurochemicals and shortduration "phasic" changes in synaptic levels (Grace, 1991, 2000; Schultz, 2007). It is possible that related mechanisms contribute to the relationship between changes in background and signal firing of OFC neurons.

OFC phasic responses to events vary with changes in the outcome of those events and are thought to contribute to a normal ability of individuals to adjust their behavior in response to changes in outcome (Schoenbaum and Roesch, 2005). Abnormalities in OFC phasic responses are further proposed to contribute to disorders such as drug addiction, where there is a diminished ability to adjust behavior in accordance with changes in behavioral outcome (Kalivas and Volkow, 2005; Stalnaker et al., 2006). Changes in background firing, like phasic responses, may play a role in mediating normal and abnormal behavioral responses to changes in response-outcome relationships.

Speaking more generally, long-lasting changes in the firing of neurons in the OFC may contribute to context-appropriate information processing and behavior (Kravitz et al., 2006) in two ways: (1) by enhancing the ratio between signal and background firing of phasic responses that are most relevant to the context and (2) by minimizing the signal:background ratio of less relevant phasic responses. In addition to modulating phasic responses in a context-dependent manner, changes in background firing rate during operant sessions may also contribute to certain forms of context-dependent learning. The long-lasting changes in background firing rates of single neurons are similar to firing rates produced by sustained low-frequency stimulation parameters that have been used to induce certain forms of synaptic depression and potentiation (Dudek and Bear, 1992; Froc and Racine, 2005; Huang and Kandel, 2005; Wohrl et al., 2007). Similar activity-dependent adaptations may occur in vivo as a result of sustained changes in background firing rate like those observed in this study. This in turn could modulate the phasic responses of individual neurons to future events, in a contextdependent manner.

\section{References}

Arieli A, Sterkin A, Grinvald A, Aertsen A (1996) Dynamics of ongoing activity: explanation of the large variability in evoked cortical responses. Science 273:1868-1871.

Boulougouris V, Dalley JW, Robbins TW (2007) Effects of orbitofrontal, infralimbic and prelimbic cortical lesions on serial spatial reversal learning in the rat. Behav Brain Res 179:219-228.

Chudasama Y, Robbins TW (2003) Dissociable contributions of the orbitofrontal and infralimbic cortex to pavlovian autoshaping and discrimination reversal learning: further evidence for the functional heterogeneity of the rodent frontal cortex. J Neurosci 23:8771-8780.

Critchley HD, Rolls ET (1996) Hunger and satiety modify the responses of olfactory and visual neurons in the primate orbitofrontal cortex. J Neurophysiol 75:1673-1686.

Dudek SM, Bear MF (1992) Homosynaptic long-term depression in area CA1 of hippocampus and effects of $N$-methyl-D-aspartate receptor blockade. Proc Natl Acad Sci USA 89:4363-4367.

Eagle DM, Baunez C, Hutcheson DM, Lehmann O, Shah AP, Robbins TW (2008) Stop-signal reaction-time task performance: role of prefrontal cortex and subthalamic nucleus. Cereb Cortex 18:178-188.

Fox MD, Snyder AZ, Zacks JM, Raichle ME (2006) Coherent spontaneous activity accounts for trial-to-trial variability in human evoked brain responses. Nat Neurosci 9:23-25.

Froc DJ, Racine RJ (2005) Interactions between LTP- and LTD-inducing stimulation in the sensorimotor cortex of the awake freely moving rat. J Neurophysiol 93:548-556.

Grace AA (1991) Phasic versus tonic dopamine release and the modulation of dopamine system responsivity: a hypothesis for the etiology of schizophrenia. Neuroscience 41:1-24.

Grace AA (2000) The tonic/phasic model of dopamine system regulation and its implications for understanding alcohol and psychostimulant craving. Addiction 95 [Suppl 2]:S119-S128.

Gutierrez R, Carmena JM, Nicolelis MA, Simon SA (2006) Orbitofrontal ensemble activity monitors licking and distinguishes among natural rewards. J Neurophysiol 95:119-133.

Hosokawa T, Kato K, Inoue M, Mikami A (2007) Neurons in the macaque orbitofrontal cortex code relative preference of both rewarding and aversive outcomes. Neurosci Res 57:434-445.

Huang YY, Kandel ER (2005) Theta frequency stimulation up-regulates the synaptic strength of the pathway from CA1 to subiculum region of hippocampus. Proc Natl Acad Sci USA 102:232-237.

Kalivas PW, Volkow ND (2005) The neural basis of addiction: a pathology of motivation and choice. Am J Psychiatry 162:1403-1413.

Kiyatkin EA, Rebec GV (1996) Dopaminergic modulation of glutamateinduced excitations of neurons in the neostriatum and nucleus accumbens of awake, unrestrained rats. J Neurophysiol 75:142-153.

Kravitz AV, Moorman DE, Simpson A, Peoples LL (2006) Session-long modulations of accumbal firing during sucrose-reinforced operant behavior. Synapse 60:420-428.

Pais-Vieira M, Lima D, Galhardo V (2007) Orbitofrontal cortex lesions disrupt risk assessment in a novel serial decision-making task for rats. Neuroscience 145:225-231.

Paxinos G, Watson C (1996) The rat brain in stereotaxic coordinates. San Diego: Academic.

Peoples LL, Cavanaugh D (2003) Differential changes in signal and background firing of accumbal neurons during cocaine self-administration. J Neurophysiol 90:993-1010.

Peoples LL, Lynch KG, Lesnock J, Gangadhar N (2004) Accumbal neural 
responses during the initiation and maintenance of intravenous cocaine self-administration. J Neurophysiol 91:314-323.

Peoples LL, Kravitz AV, Guillem K (2007) The role of accumbal hypoactivity in cocaine addiction. ScientificWorldJournal 7:22-45.

Pickens CL, Saddoris MP, Gallagher M, Holland PC (2005) Orbitofrontal lesions impair use of cue-outcome associations in a devaluation task. Behav Neurosci 119:317-322.

Roesch MR, Olson CR (2005) Neuronal activity in primate orbitofrontal cortex reflects the value of time. J Neurophysiol 94:2457-2471.

Roesch MR, Taylor AR, Schoenbaum G (2006) Encoding of timediscounted rewards in orbitofrontal cortex is independent of value representation. Neuron 51:509-520.

Roesch MR, Calu DJ, Burke KA, Schoenbaum G (2007) Should I stay or should I go? Transformation of time-discounted rewards in orbitofrontal cortex and associated brain circuits. Ann N Y Acad Sci 1104:21-34.

Rolls ET, Thorpe SJ, Boytim M, Szabo I, Perrett DI (1984) Responses of striatal neurons in the behaving monkey. 3. Effects of iontophoretically applied dopamine on normal responsiveness. Neuroscience 12:1201-1212.

Schiller D, Weiner I (2004) Lesions to the basolateral amygdala and the orbitofrontal cortex but not to the medial prefrontal cortex produce an abnormally persistent latent inhibition in rats. Neuroscience 128:15-25.

Schoenbaum G, Roesch M (2005) Orbitofrontal cortex, associative learning, and expectancies. Neuron 47:633-636.

Schoenbaum G, Chiba AA, Gallagher M (1999) Neural encoding in orbitofrontal cortex and basolateral amygdala during olfactory discrimination learning. J Neurosci 19:1876-1884.

Schoenbaum G, Nugent S, Saddoris MP, Gallagher M (2002) Teaching old rats new tricks: age-related impairments in olfactory reversal learning. Neurobiol Aging 23:555-564.
Schoenbaum G, Setlow B, Nugent SL, Saddoris MP, Gallagher M (2003) Lesions of orbitofrontal cortex and basolateral amygdala complex disrupt acquisition of odor-guided discriminations and reversals. Learn Mem 10:129-140.

Schoenbaum G, Setlow B, Saddoris MP, Gallagher M (2006) Encoding changes in orbitofrontal cortex in reversal-impaired aged rats. J Neurophysiol 95:1509-1517.

Schultz W (2007) Multiple dopamine functions at different time courses. Annu Rev Neurosci 30:259-288.

Schultz W, Tremblay L, Hollerman JR (2000) Reward processing in primate orbitofrontal cortex and basal ganglia. Cereb Cortex 10:272-284.

Simmons JM, Richmond BJ (2008) Dynamic changes in representations of preceding and upcoming reward in monkey orbitofrontal cortex. Cereb Cortex 18:93-103.

Stalnaker TA, Roesch MR, Franz TM, Burke KA, Schoenbaum G (2006) Abnormal associative encoding in orbitofrontal neurons in cocaineexperienced rats during decision-making. Eur J Neurosci 24:2643-2653.

Tait DS, Brown VJ (2007) Difficulty overcoming learned non-reward during reversal learning in rats with ibotenic acid lesions of orbital prefrontal cortex. Ann N Y Acad Sci 1121:407-420.

Tremblay L, Schultz W (1999) Relative reward preference in primate orbitofrontal cortex. Nature 398:704-708.

van Duuren E, Escamez FA, Joosten RN, Visser R, Mulder AB, Pennartz CM (2007) Neural coding of reward magnitude in the orbitofrontal cortex of the rat during a five-odor olfactory discrimination task. Learn Mem $14: 446-456$.

Wohrl R, von Haebler D, Heinemann U (2007) Low-frequency stimulation of the direct cortical input to area CA1 induces homosynaptic LTD and heterosynaptic LTP in the rat hippocampal-entorhinal cortex slice preparation. Eur J Neurosci 25:251-258. 